\title{
Computer mouse use predicts acute pain but not prolonged or chronic pain in the neck and shoulder
}

\author{
J H Andersen, ${ }^{1}$ M Harhoff, ${ }^{2}$ S Grimstrup, ${ }^{2}$ I Vilstrup, ${ }^{1}$ C F Lassen, ${ }^{3}$ L P A Brandt, ${ }^{4}$ \\ A I Kryger, ${ }^{3,5}$ E Overgaard, ${ }^{1} \mathrm{~K}$ D Hansen, ${ }^{6}$ S Mikkelsen ${ }^{3}$
}

${ }^{1}$ Department of Occupational Medicine, Herning Hospital, 7400 Herning, Denmark:

${ }^{2}$ Department of Biostatistics, University of Copenhagen,

Copenhagen, Denmark;

${ }^{3}$ Department of Occupational

Medicine, Copenhagen

University Hospital, Glostrup,

Denmark: ${ }^{4}$ Department of

Occupational and Environmental

Medicine, The University

Hospital of Odense, Odense,

Denmark; ${ }^{5}$ Department of

Occupational and Environmental

Medicine, Copenhagen

University Hospital, Bisbebjerg,

Denmark; ${ }^{6}$ Division of

Biostatistics, University of

California at Berkeley, CA, USA

Correspondence to:

Johan Hviid Andersen,

Department of Occupational

Medicine, Herning Hospital,

7400 Herning, Denmark;

hecjha@ringamt.dk

Accepted 19 July 2007

Published Online First

6 August 2007

\begin{abstract}
Background: Computer use may have an adverse effect on musculoskeletal outcomes. This study assessed the risk of neck and shoulder pain associated with objectively recorded professional computer use.
\end{abstract}

Methods: A computer programme was used to collect data on mouse and keyboard usage and weekly reports of neck and shoulder pain among 2146 technical assistants. Questionnaires were also completed at baseline and at 12 months. The three outcome measures were: (1) acute pain (measured as weekly pain); (2) prolonged pain (no or minor pain in the neck and shoulder region over four consecutive weeks followed by three consecutive weeks with a high pain score); and (3) chronic pain (reported pain or discomfort lasting more than 30 days and "quite a lot of trouble" during the past 12 months).

Results: Risk for acute neck pain and shoulder pain increased linearly by $4 \%$ and $10 \%$, respectively, for each quartile increase in weekly mouse usage time. Mouse and keyboard usage time did not predict the onset of prolonged or chronic pain in the neck or shoulder. Women had higher risks for neck and shoulder pain. Number of keystrokes and mouse clicks, length of the average activity period, and micro-pauses did not influence reports of acute or prolonged pain. A few psychosocial factors predicted the risk of prolonged pain.

Conclusions: Most computer workers have no or minor neck and shoulder pain, few experience prolonged pain, and even fewer, chronic neck and shoulder pain. Moreover, there seems to be no relationship between computer use and prolonged and chronic neck and shoulder pain.

Musculoskeletal complaints have been regarded as an adverse effect of computer use, and numerous studies have been conducted, most of them pointing to an association between neck and upper extremity pain and duration of computer use. ${ }^{1-6}$ Most of these associations have been based on cross-sectional comparisons of self-reported computer, keyboard or mouse use and self-reported pain complaints. ${ }^{7}$ A minority of studies which have used clinical outcome measures have either found too few cases with traditional medical diagnoses to perform meaningful analyses or have used diagnostics that were controversial. ${ }^{36}$ Recently, methodologies for assessing work activity during computer use have been evaluated, and activitybased recordings of computer use seem to be reliable and in agreement with measures obtained by observational techniques. ${ }^{8-11}$ A recent review called for future studies to improve our understanding of safe levels of computer use by measuring the duration of computer use in a more objective way, differentiating between mouse and keyboard use. ${ }^{12}$ In a substudy of the NUDATA (Neck and Upper extremity Disorders Among Technical Assistants) study we found that even though self-reports of mouse and keyboard use were correlated with objective recordings, participants overestimated their mouse use two to three times, and their keyboard usage up to six times when compared with the recordings. ${ }^{13}$

The aim of this study was to examine if objective measures of mouse and keyboard usage predict the onset of acute, prolonged and chronic pain in the neck and right shoulder.

\section{METHODS}

\section{Subjects}

From a cohort of 6943 computer users, which has been described in detail elsewhere, ${ }^{13-16}$ a subgroup of 2146 participants installed the software program WorkPaceRecorder (WPR), which was developed by Niche Software Ltd, New Zealand. Recordings of keyboard and mouse usage were obtained for up to 52 weeks from 1585 women (mean age 42.0 years, standard deviation (SD) 8.0) and 561 men with a mean age of 42.0 years (SD 9.0). The computer workers were mainly technical assistants $(75 \%)$. The remainder were machine technicians, secretaries, building assistants and other technicians. Most of their mouse usage was when working with computer-assisted design (CAD) tools.

\section{Measurements of keyboard and mouse usage}

From personal computers (PCs) running Microsoft Windows it is possible to obtain information on all activations of the keyboard and mouse, irrespective of the application being used. For example, keystroke count, number of single and double mouse clicks, depression of keys and mouse buttons, and software applications used can be logged. The amount of time using the keyboard and the mouse was inferred from these activations, and total keying time was defined as all keying activity occurring without non-activity intervals lasting $5 \mathrm{~s}$ or more, and total mouse usage was defined similarly. The assumptions behind the intervals were that (1) if two consecutive events on the keyboard are $5 \mathrm{~s}$ apart, it is likely that the person stopped using their fingers or holding them over the keyboard, and (2) if mouse usage events (including slight movements) are $5 \mathrm{~s}$ apart the person probably removed their hand from the mouse. The measurements for this study were aggregated into weekly parameters for usage times. Table 1 shows the overall mean exposures during the recording period among women and men. 
Parameters which were correlated with keyboard or mouse time above 0.5 were excluded from the analyses. These include total numbers of mouse clicks, single mouse clicks, keystrokes and keystrokes on the numeric pad. Quartile distribution of mouse usage was 0, 2.1, 5.2, 9.0 and $46 \mathrm{~h}$ per week. Quartiles for keyboard use were $0,0.4,0.9,1.7$ and 22 h per week.

Recording of computer activity among cohort members only was secured by a personal log-on to the WPR programme.

\section{Outcome measures}

Each Friday participants were prompted to answer an on-screen complaint questionnaire as a part of the WPR monitoring procedure. If the participant was not using the computer on Friday, the software prompted next time they did use the computer until Tuesday in the following week for complaints in the previous week. The questionnaire asks the participants to rate their degree of pain within the last 7 days on a scale from 0 to 7 with the verbal anchors: no pain, very mild pain, mild pain, mild to moderate pain, moderate pain, moderate to severe pain, severe pain and very severe pain. Levels 5, 6 and 7 were collapsed in the analyses giving six levels of pain on an ordinal scale from 0 . For each region a different image was displayed highlighting the body area of interest. The weekly levels of neck and right shoulder pain constitute the outcome measures: (1) acute neck pain level and (2) acute shoulder pain level. If there were missing responses to the weekly pain assessments, imputed values were calculated if there were values in the week before and in the week after the missing value. If the week before and the week after were the same, the missing value was given that value. In the case where there was one category difference between the week before and the week after the missing value, the missing value was randomly selected from the week before or the week after a missing value. Differences of more than two categories were not imputed and not included in the analysis.

In an attempt to capture participant's transition from no or minor pain to a more pronounced pain intensity, the computer programme identified incident prolonged pain cases defined as a participant averaging 2.5 or less in the neck and shoulder region over four consecutive weeks followed by three consecutive weeks with a pain score average of 4 or more in that region. The two incident pain outcomes constitute the third and fourth outcome measures: (3) incident "prolonged neck pain" and (4) incident "prolonged shoulder pain".

At 1-year follow-up, participants completed a postal questionnaire, and on the basis of answers to questions relating to neck and shoulder complaints, two measures of chronic pain were constructed: participants who reported pain or discomfort lasting more than 30 days and had experienced at least "quite a lot of trouble" during the past 12 months but had been free of pain above 3 in that region at baseline. Outcome 5 is thus "chronic neck pain", and outcome 6 "chronic shoulder pain". From personal date logs we restricted the analysis to recordings prior to completing the 1-year questionnaire.

\section{Background variables}

From a baseline questionnaire, a series of covariates regarding physical, psychosocial and individual characteristics was included in the analyses. These characteristics have been described in detail elsewhere. ${ }^{6}{ }^{13-16}$ In short, these variables were individual factors (gender, age, type A behaviour, negative affectivity, private social support, medical diseases with potential to influence neck and upper limb pain status, and accidents involving injury of the neck or shoulder), psychosocial work environment factors (job demands, job control, social support, and time pressure at work) and ergonomic factors (abnormal position of mouse or keyboard, lack of arm/wrist support, height of screen, and adjustable work desk and chair). Seniority was assessed by the length of time participants had used a computer at work to the same extent as currently, divided into less than 3 years, 4-7 years, 8-10 years and more than 10 years.

\section{Statistical analyses}

The relationship between neck pain and right shoulder pain, and the independent variables was analysed in three different ways.

Firstly, acute neck and shoulder pain were analysed by autoregressive ordinal regression analyses with the recorded exposure variables from the week preceding the reports of pain. Earlier pain reporting was included in the models as the mean over the four preceding weeks based on the correlation data structure of pain reporting. Restricted cubic splines were used to examine the relationships. ${ }^{17}$ The basic point in the models was an attempt to isolate the direct influence of mouse and keyboard usage on the pain level, adjusted for correlations in individuals and feedback effects. The aim of these analyses is to estimate an intra-individual effect of the exposure on the following week's pain level. The analyses were performed with R. We further used a GEE approach in a simple ordinal regression model to compare the results obtained from the autoregressive models. The results were very similar, and we report the results from the autoregressive analysis in this paper because we find them most suitable with this kind of data correlation structure. Fixed covariates from baseline were included in all models.

The second set of analyses involved analysing incident prolonged pain by Cox regression. Covariates from baseline on individual, psychosocial and ergonomic risk factors were included in all the models, and the interquartile ranges of weekly mouse and keyboard use preceding the occurrence of prolonged pain were tested. None of the interaction terms

Table 1 Distributional characteristics of the recorded exposure variables from WorkPaceRecorder among men and women

\begin{tabular}{|c|c|c|c|c|c|c|}
\hline \multirow[b]{2}{*}{ Exposure variables } & \multicolumn{3}{|c|}{ Female $(\mathrm{n}=78$ 171-78 177) } & \multicolumn{3}{|c|}{ Male ( $n=27$ 669-27 671) } \\
\hline & Mean (SD) & Min & Max & Mean (SD) & Min & Max \\
\hline Computer use in hours per week & $9.2(6.2)$ & 0 & 57.8 & $9.2(6.5)$ & 0 & 63.9 \\
\hline Mouse usage in hours per week & $6.1(4.9)$ & 0 & 42.6 & $5.8(4.8)$ & 0 & 45.7 \\
\hline Keyboard usage in hours per week & $1.3(1.3)$ & 0 & 15.6 & $1.3(1.5)$ & 0 & 21 \\
\hline Mouse clicks (per 25 clicks per minute) & $1.0(0.3)$ & 0.1 & 3.8 & $0.9(0.3)$ & 0.1 & 3.5 \\
\hline Keystrokes (per 100 per minute) & $1.5(0.2)$ & 0.6 & 4.6 & $1.4(0.2)$ & 0.6 & 4.1 \\
\hline Average period of active mouse use (per $10 \mathrm{~min}$ ) & $0.3(0.2)$ & 0 & 6.1 & $0.2(0.2)$ & 0 & 6.2 \\
\hline Average period of active mouse breaks (per minute) & $0.7(0.3)$ & 0 & 3.4 & $0.8(0.3)$ & 0 & 3.3 \\
\hline Average period of active keyboard use (per $2 \mathrm{~min}$ ) & $0.1(0.1)$ & 0 & 4.2 & $0.1(0.1)$ & 0 & 2.3 \\
\hline Average period of active keyboard breaks (per minute) & $0.6(0.5)$ & 0 & 5.3 & $0.6(0.4)$ & 0 & 4.2 \\
\hline
\end{tabular}


between any of the recorded exposure variables contributed significantly to any of models. Analyses were conducted using the Stata statistical package (v 9.0; Stata, College Station, TX).

Thirdly, for the purpose of analysis of chronic neck and shoulder pain, we used a logistic regression model to analyse the crosssectional associations between registered cumulative mouse and keyboard use in the year preceding the follow-up questionnaire. In this model, mouse and keyboard usage was summed across the 52 weeks to give a cumulative number of hours working with the mouse and keyboard within the year preceding the report of chronic neck and shoulder pain in the 1-year follow-up questionnaire. The quartiles for hours per year with a mouse were 0 , 160, 273, 413 and $1590 \mathrm{~h}$ per year. For a keyboard the quartiles were $0,30,50,80$, and $550 \mathrm{~h}$ per year. Due to a shortage of chronic pain cases, we only included mouse and keyboard usage time, age, gender and seniority in these models.

\section{RESULTS}

The 2146 participants were recruited from an original cohort of 6943 technicians. There were several reasons why some subjects were not included in this part of the study: lack of an employer's approval for installing the software programme, participants using more than one computer at work simultaneously, working with platforms other than Windows 95/98/ NT4, and of course the participants refraining from installing the programme for other reasons. Among the participants who installed WorkPaceRecorder, 11.1\% graded their neck pain within the last 7 days as 4 or more on the pain scale from 0 to 7 , and $8.0 \%$ graded their shoulder pain to be 4 or more. The same percentages for the whole cohort were $10.7 \%$ and $7.7 \%$. Self-reported mouse use was a little higher in the WPR group with a mean weekly usage of 13.4 vs $11.4 \mathrm{~h}$ per week in the full cohort. Keyboard time was $8.8 \mathrm{~h}$ per week in both groups. More women installed the programme: $73.8 \%$ vs $62.6 \%$ in the baseline cohort.

WPR data files were returned by 2146 participants, and 105848 weekly observations were obtained. Overall, 84\% $(\mathrm{n}=1795)$ of respondents returned data files for 52 weeks, and $95 \%$ of the data files included at least 30 weeks. A total of 90388 weekly questionnaires were completed (85\%).

\section{Acute pain}

Table 2 shows the percentage of participants reporting pain in the neck and the right shoulder during the recording period. Most participants did not experience neck and shoulder pain. However, $6.3 \%$ of males and $9.1 \%$ of females experienced severe pain in the neck in any 1 week during the study period, and $9.8 \%$ males and $10.2 \%$ females reported severe shoulder pain. Figure 1 illustrates a linear association between mouse usage time and acute neck and shoulder pain with significant differences from a zero effect. The effect on neck pain corresponds to a $0.6 \%$ increase in risk for a $1 \mathrm{~h}$ increase in weekly usage, and for shoulder pain the risk increase per hour is $2 \%$. In table 3 the risk increase is shown for an increase in the interquartile range of mouse usage with an odds ratio (OR) for acute neck pain of 1.04 (95\% CI 1.00 to 1.09), and for acute shoulder pain an OR of 1.10 (95\% CI 1.05 to 1.16). Figure 1 also illustrates a non-effect of keyboard usage on both acute neck and shoulder pain. From table 3 it can also be seen that none of the variables for mouse and keyboard speed, micro-pauses or length of activity periods had any main effects on acute pain. Women had an increased risk for acute neck pain (OR 1.21, 95\% CI 1.16 to 1.27 ) and for acute shoulder pain (OR 1.22, 95\% CI 1.16 to 1.28$)$.

\section{Prolonged and chronic pain}

Table 4 shows the incidence of prolonged and chronic neck and shoulder pain for men and women. Onset of prolonged neck pain was seen in 302 participants and prolonged shoulder pain occurred in 227; the incidence rates were 0.0036 for prolonged neck pain and 0.0026 for prolonged shoulder pain. Few participants fulfilled our criteria for incident chronic pain at 1year follow-up. A total of $1.75 \%$ of males and $2.13 \%$ of females reported new chronic neck pain during 1 year, and the corresponding percentages for chronic shoulder pain were $1.89 \%$ of males and $2.5 \%$ of females. Table 3 reveals that association between mouse usage time and acute pain does not hold for prolonged pain in the neck or shoulder. None of the recorded computer use exposure variables had an effect on prolonged pain in the neck and shoulder. Females showed an increased risk of around $80 \%$ for prolonged pain. Among the potential confounders included, only a few were significant besides gender. Overall dissatisfaction with the physical lay-out of the work station was associated with prolonged neck pain (OR 1.76, 95\% CI 1.22 to 2.55). Low social support in the work place was associated with neck pain (OR 1.56, 95\% CI 1.21 to 2.03). Prolonged shoulder pain was associated with the combination of high job demands and low job control (OR $2.30,95 \%$ CI 1.24 to 4.28 ). None of the other fixed variables from baseline on ergonomic and individual risk factors were associated significantly with prolonged neck and shoulder pain.

Table 5 shows associations between cumulative mouse and keyboard usage time and the risk for chronic neck and shoulder pain. Time with mouse or keyboard did not predict chronic pain at 1-year follow-up. Neither age nor seniority was significantly associated with chronic shoulder pain, but a trend was seen for more neck pain with greater seniority ( $p=0.04$ for trend).

\section{DISCUSSION}

This is the first prospective study of neck and shoulder pain among computer users which benefits from continuously recorded objective computer usage, weekly measures of pain and the opportunity to identify incident cases with prolonged pain from a definition included in the computer programme.

In this study we found that most participants reported no or minor pain problems in the neck and shoulder. The prevalence of weekly severe pain was low, the incidence of prolonged neck pain was $0.31 \%$, and the incidence of prolonged shoulder pain was $0.23 \%$. Analyses of risk factors related to computer work showed that for every quartile increase in weekly mouse usage, the risk for acute neck pain increased by $4 \%$ and the risk for acute shoulder pain by $10 \%$. Analyses of time with mouse and keyboard showed no threshold level for the time variables. Exclusion of the $5 \%$ of subjects with zero values for mouse and keyboard usage some time in the 52-week period of recording did not change the linear relationship or the percentages of increase for every quartile of increase in mouse or keyboard usage. None of the other recorded variables such as typing/ clicking speed, average activity period lengths or micro-pauses, influenced the results. Prolonged and chronic neck and shoulder pain were not related to computer use, neither to mouse use nor keyboard use.

Our specific definition of incident prolonged pain is open to discussion. The participants should fulfil the option of four consecutive weeks with no or minor pain followed by 3 weeks with more severe pain. There are probably other ways to advance from minor pain to severe pain. We tested if any combination of long-lasting severe pain for three, four or six consecutive weeks was associated with our exposure variables in 

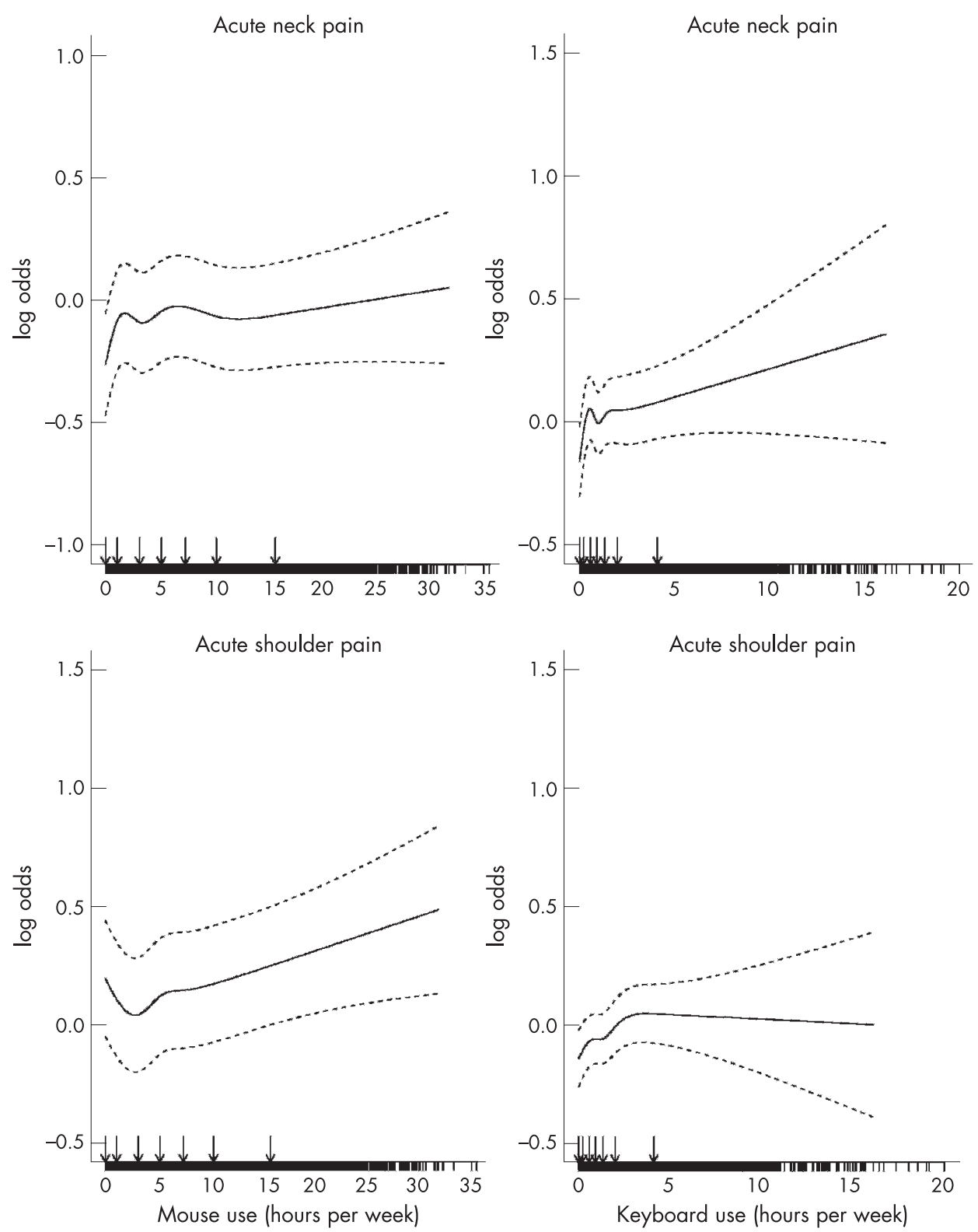

Figure 1 Adjusted log odds with 95\% confidence intervals for acute neck and shoulder pain in relation to mouse (left) and keyboard use (right) in the preceding week. The vertical arrows show the knots used for modelling with restricted cubic splines in the autoregressive ordinal regression models. The curves show the linear association between mouse and keyboard usage with all other variables at a fixed level.

Table 2 Overall percentages and within percentages of acute neck and shoulder pain among 2146 computer users with weekly recordings for 52 weeks

\begin{tabular}{|c|c|c|c|c|c|c|c|c|}
\hline & \multicolumn{4}{|c|}{ Acute neck pain } & \multicolumn{4}{|c|}{ Acute shoulder pain } \\
\hline & \multicolumn{2}{|c|}{ Males $(n=561)$} & \multicolumn{2}{|c|}{ Females $(n=1585)$} & \multicolumn{2}{|c|}{ Males $(n=561)$} & \multicolumn{2}{|c|}{ Females $(n=1585)$} \\
\hline & $\begin{array}{l}\text { Overall \% } \\
(\mathrm{n}=24878)\end{array}$ & $\begin{array}{l}\text { Within \% } \\
(n=1737)\end{array}$ & $\begin{array}{l}\text { Overall \% } \\
\text { (n=69 957) }\end{array}$ & $\begin{array}{l}\text { Within \% } \\
(\mathrm{n}=5775)\end{array}$ & $\begin{array}{l}\text { Overall \% } \\
(n=24851)\end{array}$ & $\begin{array}{l}\text { Within \% } \\
(n=1533)\end{array}$ & $\begin{array}{l}\text { Overall \% } \\
(n=70060)\end{array}$ & $\begin{array}{l}\text { Within \% } \\
(\mathrm{n}=5240)\end{array}$ \\
\hline No pain & 65.3 & 69.6 & 54.3 & 59.0 & 70.5 & 74.7 & 60.2 & 65.0 \\
\hline Very mild pain & 16.3 & 22.6 & 19.9 & 24.5 & 14.2 & 22.0 & 17.5 & 22.7 \\
\hline Mild pain & 10.2 & 16.9 & 13.1 & 17.6 & 8.5 & 16.8 & 11.6 & 17.4 \\
\hline Severe pain & 1.2 & 6.3 & 2.4 & 9.1 & 1.1 & 9.8 & 2.0 & 10.2 \\
\hline
\end{tabular}


Table 3 Risk estimates for acute* and prolonged pain $\dagger$ in the neck and right shoulder in relation to recorded exposure variables

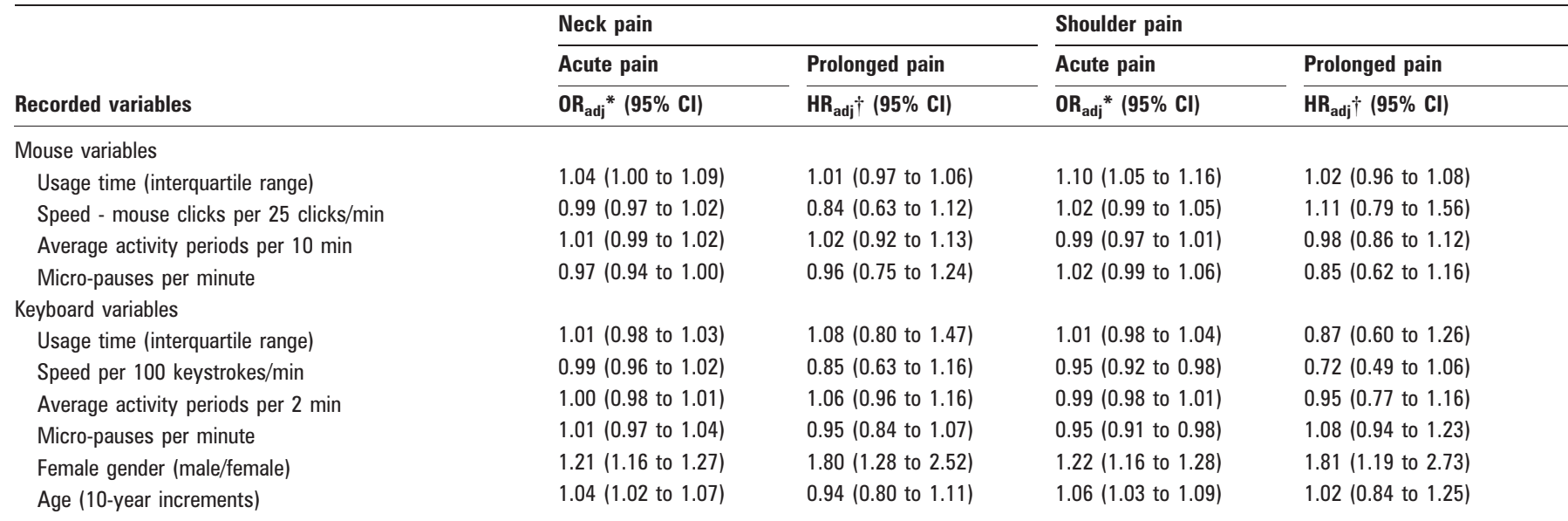

${ }^{*}$ Acute pain with odds ratios (OR) obtained by autoregressive ordinal logistic regression analysis; †prolonged pain with hazard ratios (HR) obtained by Cox regression analysis with mutual adjustments for fixed covariates.

a different way from our definition of prolonged pain. There were no such indications (results not shown).

An explanation for an effect on acute pain, but not on prolonged or chronic pain, could be reverse causation in that the level of pain could influence the amount of mouse and keyboard usage in the subsequent week. We tested whether level of any musculoskeletal pain predicted the following week's usage time using a random effects regression model and found no effects ( $p=0.49$ for men and $p=0.30$ for women).

Some of the fixed covariates from the baseline assessment were risk factors for neck and shoulder pain. The most consistent risk factor was female gender, but being unsatisfied with the physical lay-out of the workplace was also associated with neck pain even though none of the specific ergonomic risk factors showed any contribution to the models. Asking about satisfaction with the physical lay-out of the workplace was meant to capture other ergonomic factors besides those actually asked about, but it could also serve to indicate overall dissatisfaction with the work environment, tasks or other factors. Low social support at work was a risk factor for neck pain but not for shoulder pain, and high job strain was predictive of prolonged shoulder pain.

Table 4 Incidence of prolonged (per week) and chronic (1-year) pain in the neck and right shoulder within 1 year of recordings

\begin{tabular}{|c|c|c|c|c|}
\hline Outcome & Subjects & Cases & $\begin{array}{l}\text { Time } \\
\text { at risk } \\
\text { (weeks) }\end{array}$ & $\begin{array}{l}\text { Incidence } \\
\text { rate per week } \\
\text { /1-year } \\
\text { incidence }(\%)\end{array}$ \\
\hline \multicolumn{5}{|c|}{ Prolonged neck pain } \\
\hline Males & 561 & 54 & 26352 & 0.00205 \\
\hline Females & 1585 & 248 & 72196 & 0.00344 \\
\hline Total & 2146 & 302 & 98548 & 0.00306 \\
\hline \multicolumn{5}{|c|}{ Chronic neck pain } \\
\hline Males & 458 & 8 & & $1.75 \%$ \\
\hline Females & 1172 & 25 & & $2.13 \%$ \\
\hline Total & 1630 & 33 & & $2.02 \%$ \\
\hline \multicolumn{5}{|c|}{ Prolonged shoulder pain } \\
\hline Males & 561 & 39 & 26731 & 0.00146 \\
\hline Female & 1585 & 188 & 73649 & 0.00255 \\
\hline Total & 2146 & 227 & 100380 & 0.00226 \\
\hline \multicolumn{5}{|c|}{ Chronic shoulder pain } \\
\hline Males & 477 & 9 & & $1.89 \%$ \\
\hline Females & 1239 & 31 & & $2.50 \%$ \\
\hline Total & 1716 & 40 & & $2.33 \%$ \\
\hline
\end{tabular}

An explanation for the low occurrence of chronic pain in this study could be selection out of the cohort because of neck and shoulder pain. Our data do not support this. The levels of musculoskeletal pain were no different among the 6943 subjects at baseline, the 5658 who completed 1-year follow-up, the 1335 drop-outs from baseline to 1-year follow-up, and the 2146 who participated in this study. Furthermore, overall sickness absence for more than 2 weeks, obtained from the Danish central register, did not reveal higher sickness absence among non-responders at baseline or at follow-up, which strongly points against healthy worker selection in this group of computer workers.

One interesting aspect of our study was that it was possible to conduct a large scale epidemiological study using objective measures of exposure. The participation rate with respect to installing and returning WPR data was rather low (31\%), however, but participants who returned data did not differ very much from those who did not with respect to questionnaire data on computer work and musculoskeletal pain. The participants in this study reported slightly higher weekly mouse usage than the full cohort and there was an over-representation of women. So this study included more heavy female mouse users than the original cohort, but we do not think that the associations between computer use and pain-related variables would be severely biased by this. Considering that the study covered more than 3000 work places with different software platforms and policies for software installation, we find the

Table 5 Risk factors* for chronic neck and shoulder pain at 1-year follow-up

\begin{tabular}{|c|c|c|}
\hline & $\begin{array}{l}\text { Chronic neck pain } \\
\text { OR }(95 \% \mathrm{CI})\end{array}$ & $\begin{array}{l}\text { Chronic shoulder } \\
\text { pain OR }(95 \% \mathrm{CI})\end{array}$ \\
\hline $\begin{array}{l}\text { Mouse usage, quartiles of } \\
\text { hours per year }\end{array}$ & $0.77(0.55$ to 1.07$)$ & $1.11(0.86$ to 1.44$)$ \\
\hline $\begin{array}{l}\text { Keyboard usage, quartiles } \\
\text { of hours per year }\end{array}$ & $1.05(0.74$ to 1.51$)$ & 0.91 (0.68 to 1.21 ) \\
\hline Male/female & $1.43(0.62$ to 3.30$)$ & 1.20 (0.55 to 2.58$)$ \\
\hline \multicolumn{3}{|l|}{ Seniority } \\
\hline$\leqslant 3$ years $(n=529)$ & 1.00 (ref) & 1.00 (ref) \\
\hline $4-7$ years $(n=780)$ & 1.06 (0.36 to 3.07$)$ & $0.58(0.24$ to 1.41$)$ \\
\hline $8-10$ years $(n=442)$ & $1.88(0.65$ to 5.44$)$ & $0.53(0.18$ to 1.54$)$ \\
\hline$>10$ years $(n=375)$ & $2.53(0.84$ to 7.56$)$ & 1.08 (0.43 to 2.69 ) \\
\hline Age, 10 -year increments & 0.85 (0.52 to 1.39 ) & 0.89 (0.55 to 1.45$)$ \\
\hline
\end{tabular}




\section{Main messages}

- Most computer workers have no or minor neck and shoulder pain complaints, few experience prolonged pain, and even fewer, chronic neck and shoulder pain.

- Objectively measured mouse and keyboard activities were positively associated with acute neck and shoulder pain but were not associated with prolonged or chronic neck and shoulder pain.

- Earlier results from this cohort based on self-reports of mouse and keyboard use could be biased.

participation rate quite satisfactory. Much higher participation rates in large epidemiological studies using electronic activity recordings can probably be expected in studies concentrated in a few large companies.

In general, the cohort members were mouse users more than they were keyboard users. The mean mouse usage was around $6 \mathrm{~h}$ per week, but there was a large variation with $10 \%$ of the weeks without any mouse use and around $10 \%$ with more than $15 \mathrm{~h}$ per week. Total computer use was $9.2 \mathrm{~h}$ per week and was strongly correlated with mouse use with a correlation coefficient of 0.94 . Keyboard usage was at a low level, so the results for keyboard users should be cautiously interpreted and may not hold for heavy keyboard users. The maximum keyboard use in our cohort was around $20 \mathrm{~h}$ per week.

Participation in the study and answering questions about pain every week might induce heightened awareness of pain and aggravation of any minor sensations, but the recorded levels of computer activity were not known to the participants. In addition, the length of the monitoring time period makes it unlikely that the risk estimates for mouse and keyboard usage were flawed by information bias.

Limitations of the study include the fixed covariates, which were self-assessed only at baseline, and we do not know the exact extent to which ergonomic or psychosocial work place factors changed during the recording period. The results from other analyses in the NUDATA study do not point to differences in risk estimates for ergonomic risk factors when comparing those who made changes in how they carried out their job and those who did not. ${ }^{6}{ }^{13}$

Findings from the NUDATA study on neck and shoulder pain in relation to self-reported usage times for mouse and keyboard have been previously reported. ${ }^{6}$ This study confirms the linear relationship between mouse usage time and acute pain, but it strongly questions the existence of any relationship between computer use and prolonged and chronic pain. Concerning 1year incident chronic pain, we found an effect of mouse use on shoulder pain when exposure was based on self-reports, but this association disappears in this study where we have included objective measurements of mouse use. This contradiction raises the possibility of severe information bias, probably associated with the setting and launching of studies of computer use and musculoskeletal pain. Even though the NUDATA study was launched as a general study of work and health among technicians, discussions among technicians and writings in the media have centred on lay understandings of the "mouse arm", and under these circumstances the possibility for information bias is huge. In earlier studies, clinical outcomes are rare, and existing evidence for a detrimental effect on the neck and shoulder of computer use is sparse. From the NUDATA study we can conclude that most computer workers have no or minor

\section{Policy implications}

- The common opinion that computer use has an adverse health effect is questionable.

- Future research on the relationship between computer work and adverse health effects should utilise objective assessments of mouse and keyboard use.

pain complaints, few experience prolonged pain, and even fewer, chronic pain. Moreover, there seems to be no relationship between computer use and our measures of prolonged and chronic neck and shoulder pain. A recent review ${ }^{12}$ found stronger risk estimates for the hand-arm region than for the neck-shoulder region based on the few prospective studies identified, and the possibility for hand-arm disorders should be further studied. The results from the NUDATA study of distal arm pain will be reported in a separate paper.

Acknowledgements: We thank the Danish Association of Professional Technicians for useful assistance, and we especially thank the study participants for their time and effort.

Funding: This study was supported by grant 9801292 from the Danish Medical Research Council and grant 20000010486 from the Danish Ministry of Employment, National Work Environment Authority.

Competing interests: None.

\section{REFERENCES}

1. Punnett L, Bergqvist U. Visual display unit work and upper extremity musculoskeletal disorders: a review of epidemiological findings. Arbete och Halsa 16. Solna: Arbetslivsinstitutet, 1997

2. Blatter BM, Bongers PM. Duration of computer use and mouse use in relation to musculoskeletal disorders of the neck and upper limb. Int J Ind Ergon 2002;30:295306.

3. Gerr F, Marcus M, Ensor C, et al. A prospective study of computer users: I. Study design and incidence of musculoskeletal symptoms and disorders. Am J Ind Med 2002;41(4):221-35

4. Korhonen T, Ketola R, Toivonen R, et al. Work related and individual predictors for incident neck pain among office employees working with video display units. Occup Environ Med 2003;60(7):475-82.

5. Jensen C. Development of neck and hand-wrist symptoms in relation to duration of computer use at work. Scand J Work Environ Health 2003;29(3):197-205.

6. Brandt LPA, Andersen JH, Lassen CF, et al. Neck and shoulder symptoms and disorders in a Danish cohort of 6943 computer workers. Scand J Work Environ Health 2004;30:399-409.

7. Gerr F, Marcus M, Monteilh C. Epidemiology of musculoskeletal disorders among computer users: lesson learned from the role of posture and keyboard use. J Electromyogr Kinesiol 2004;14(1):25-31

8. Homan MM, Armstrong TJ. Evaluation of three methodologies for assessing work activity during computer use. AlHA J 2003;64(1):48-55.

9. Blangsted AK, Hansen K, Jensen $C$. Validation of a commercial software package for quantification of computer use. Int $J$ Ind Ergon 2004;34:237-41.

10. Heinrich J, Blatter BM, Bongers PM. A comparison of methods for the assessment of postural load and duration of computer use. Occup Environ Med 2004;61(12): 1027-31.

11. Douwes M, de Kraker H, Blatter BM. Validity of two methods to assess computer use: self-report by questionnaire and computer use software. Int $\mathrm{J}$ Ind Ergon 2007:37:425-31.

12. Ljemker S, Huysmans M, Blatter BM, et al. Should office workers spend fewer hours at their computer? A systematic review of the literature. Occup Environ Med 2007:64(4):211-22

13. Mikkelsen S, Vilstrup I, Lassen CF, et al. Validity of questionnaire self-reports on computer, mouse and keyboard usage during a 4 week period. Occup Environ Med 2007:64:541-7.

14. Lassen CF, Mikkelsen S, Kryger A, et al. Elbow and wrist/hand symptoms among 6943 computer operators. A 1-year follow-up study (the NUDATA study). Am J Ind Med 2004;46:521-33

15. Kryger AI, Andersen JH, Lassen CF, et al. Is intensive use of the arms an occupational hazard among computer users? From the NUDATA study. Occup Environ Med 2003;60(11):e14

16. Andersen JH, Thomsen JF, Overgaard E, et al. Computer use and carpal tunnel syndrome: a 1-year follow-up study. JAMA 2003;289(22):2963-9.

17. Diggle PJ, Heagerty P, Liang KY, et al. Analysis of longitudinal data. Oxford: Oxford University Press, 2002 